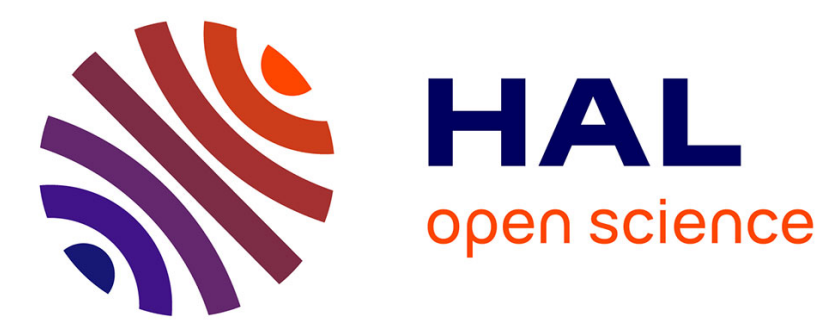

\title{
Tensile damage response from discrete element virtual testing
}

Arnaud Delaplace

\section{To cite this version:}

Arnaud Delaplace. Tensile damage response from discrete element virtual testing. Geomechanics and Geoengineering, 2009, 4 (1), pp.79-89. 10.1080/17486020902767339 . hal-00412937

\section{HAL Id: hal-00412937 https://hal.science/hal-00412937}

Submitted on 2 Sep 2009

HAL is a multi-disciplinary open access archive for the deposit and dissemination of scientific research documents, whether they are published or not. The documents may come from teaching and research institutions in France or abroad, or from public or private research centers.
L'archive ouverte pluridisciplinaire HAL, est destinée au dépôt et à la diffusion de documents scientifiques de niveau recherche, publiés ou non, émanant des établissements d'enseignement et de recherche français ou étrangers, des laboratoires publics ou privés. 


\title{
Tensile damage response from discrete element virtual testing
}

\author{
A. DELAPLACE \\ LMT-Cachan, ENS Cachan/CNRS/UPMC/PRES UniverSud Paris \\ 61, avenue du Président Wilson \\ 94235 Cachan, France
}

\begin{abstract}
Depending on the loading conditions on brittle materials, damage can generally not be reduced to a simple scalar. Microcrack orientation affects the stiffness in a preferential direction perpendicular to the crack lips. Taking into account the damage anisotropy in phenomenological models is a possible option, but the identification of the corresponding models with respect to damage anisotropy is not an easy task, usually because of the limited number of experimental results. Handling and performing tensile experiments on damaged samples made of quasi-brittle heterogeneous material is quite delicate. Then, one can consider virtual testing for the identification of specific properties. We propose in the present work such a protocol, based on the use of discrete element models and codes as a virtual testing machine. Discrete models are based on a material description at the microscopic scale by an assembly of particles. Cracks (and cracks orientations) are naturally described by broken connections between particles. After a brief description of the chosen model, a cross-identification procedure is proposed for the determination of an hydrostatic sensitivity parameter of an anisotropic damage model. A second application deals with the description of the evolution of the strength envelope under different isotropic and anisotropic damage states. These examples show the interest of virtual testing by discrete modeling for material behavior characterization at fine scale.
\end{abstract}

Keywords: discrete model; damage; anisotropy; brittle materials; virtual testing

\section{Introduction}

Damage anisotropy becomes nowadays an important concern for structures durability studies. A simple calculation of crack density in the material is no more sufficient when dealing with flow through a cracked wall, or with sliding dissipation under a cyclic loading: one needs to have access to crack orientation, crack opening. Considering phenomenological model for describing large scale structure behavior, two main kinds of approaches can be used to determine crack orientations: a fracture mechanics crack description using XFEM approach for example [Moes et al., 1999, Wells and Sluys, 2000], or a tensorial damage mechanics description [Cordebois and Sidoroff, 1982, Murakami, 1988, Lemaitre and Desmorat, 2005], instead of a simpler scalar (isotropic) damage description. But the parameter identification of such models is often uneasy because of the lack of experimental results. Performing reliable and reproductive tests on damage samples made of brittle material is difficult, especially under tensile loading or more generally under positive hydrostatic stresses. Note that recent experimental measurement devices such as digital image correlation techniques can help to perform such delicate tests 
[Sutton et al., 2000, Fayolle et al., 2007]. An alternative for identification procedure is the concept of "virtual testing" or "cross-identification", in which a numerical discrete or damage model is preferred (see for example [Garboczi et al., 2004, Sun et al., 2007, Gonzlez and LLorca, 2007] for recent applications). Such an approach cannot obviously replace a full experimental process but it can help for identifying specific properties.

One proposes in this work to use a discrete element model [Cundall and Strack, 1979, Herrmann and Roux, 198 Schlangen and Van Mier, 1992] as a "virtual testing" machine. The model is based on a Voronoi particles assembly for describing material microstructure. Material heterogeneity is naturally taken into account through the mesh randomness, and a non linear material behavior is obtained by considering brittle links between particles. Due to its natural and explicit description of cracks [Bolander and Saito, 1998, D'Addetta et al., 2002, Potyondy and Cundall, 2004, Delaplace and Ibrahimbegovic, 2006], this kind of model is powerful for describing quasi-brittle material behavior, in particular under tensile loading.

Once the description of the discrete element model made, one uses the model for the characterization of material behavior under anisotropic damage states. A first application is devoted to the cross-identification of a 3D anisotropic damage model (see [Desmorat and Otin, 2007] for other damage cross-identification procedures). The strength of the model is that the dissymmetry tension/compression is due to the loading induced anisotropy so that six parameters (including two parameters for elasticity) are sufficient to describe both the tension and compression responses of concrete. The anisotropic damage pattern of microcracks orthogonal to the stress in tension and parallel to the stress in compression is represented. But one of the parameters introduced, the parameter $\eta$ of sensitivity to hydrostatic stresses, is tricky to identify because it only acts on tensile hydrostatic stresses, or in an equivalent manner on the bulk modulus. The discrete model is then used to identify this specific parameter. A procedure based on a succession of loading/unloading tests with increasing damage states is performed on cube samples with different boundary conditions. A second application concerns the evolution of the failure envelope in biaxial tensile loading. Different studies [Kupfer and Hilsdorf, 1969, Lee et al., 2004] have already proposed an experimental determination of this function but only for an initial undamaged state. Determining the failure envelope for pre-damaged samples, under isotropic or anisotropic damage, is more difficult. Again, the discrete model is used opportunely for this purpose. A parallelepiped sample, similar to the representative volume element tested in the experimental studies, is broken under different initial damage states. The failure envelope is determined for the different pre-damaged states.

\section{Discrete model}

In the considered discrete model, the material is described as a Voronoi particles assembly, representative of the material heterogeneity. A grid support is used for generated Voronoi particles nuclei [Moukarzel and Herrmann, 1992] in order to easily control mesh variability and to simplify the application of boundary conditions. Figure 1 presents two examples of meshes, the first one in 2D and the second one in 3D. Basically, two kinds of interactions are considered: cohesive and contact forces. Because our study focuses only on tension loading, we consider next only cohesive forces.

\subsection{Cohesive forces}

Interaction between particles is limited to cohesive forces. In 3D, each particle has six degrees of freedom (three translations and three rotations) and a $12 \times 12$ local stiffness matrix is adequate to represent the complete interaction. Following [Schlangen and Garboczi, 1997, Van Mier et al., 2002], an Euler-Bernoulli beam matrix is used in the model to connect each pair of neighboring particles 

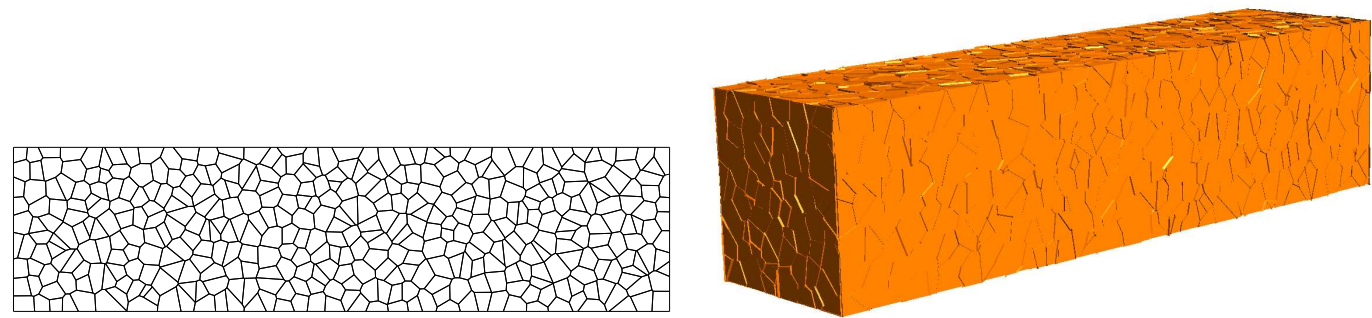

Figure 1: Example of a 2D mesh (top) and a 3D one (bottom).

$i$ and $j$. Such matrix depends on four beam parameters: the Young modulus $E^{b}$ (chosen equal for all beams), the area $A_{i j}^{b}$, the length $\ell_{i j}^{b}$ and the moment of inertia $I_{i j}^{b}$. Mesh geometry fixes two parameters:

- $\ell_{i j}^{b}$ is the distance between the two particle centers,

- $A_{i j}^{b}$ is the area of the common facet (a convex polygon for the Voronoi tesselation) of the two particles $i$ and $j$.

The two other parameters $E_{b}$ and $I_{i j}^{b}$ are identified from material elastic parameters, the Young modulus $E$ and the Poisson coefficient $\nu . E_{b}$ is easily identified as it is directly proportional to $E$ and it has no effect on $\nu$. The moment of inertia can be estimated from the curve plotted in figure 2: it shows the evolution of $\nu$ versus the adimensioned (with respect to the equivalent circular moment of inertia) moment of inertia $\alpha$.

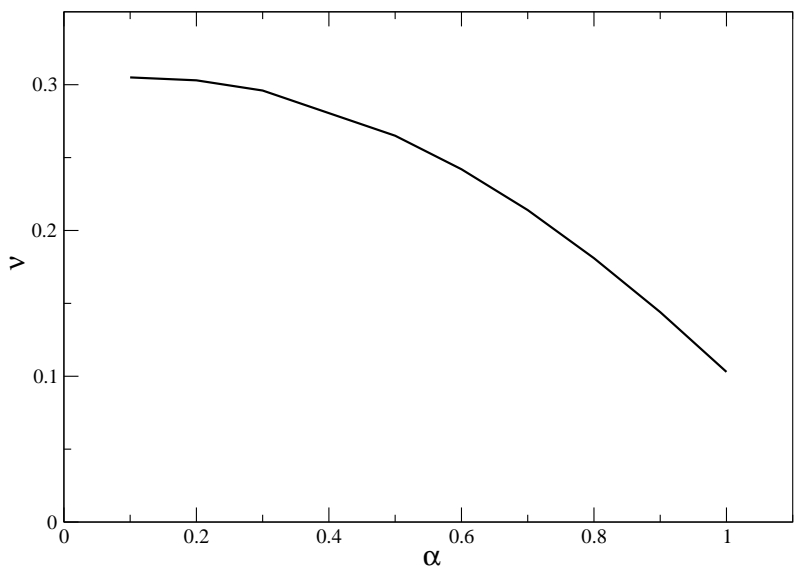

Figure 2: Evolution of elastic material parameter $\nu$ with respect to beam parameter $\alpha$.

\subsection{Nonlinear behaviour}

Damage evolution of the material is rendered by considering a perfectly elastic behavior for the beams. The breaking criteria for a beam $i j$ depends on its strain $\varepsilon_{i j}$ and on rotations of both particles $i$ and $j$ :

$$
P_{i j}=\left(\frac{\varepsilon_{i j}}{\varepsilon^{c r}}\right)^{2}+\left(\frac{\max \left(\left|\theta_{i}\right|,\left|\theta_{j}\right|\right)}{\theta^{c r}}\right) \geq 1
$$


$\theta_{i}$ and $\theta_{j}$ are the maximum rotation value of particles $i$ and $j$. Parameters $\varepsilon^{c r}$ and $\theta^{c r}$ control the nonlinear evolution of the model. The first variable acts mainly on tensile behavior as the second one acts on compressive behavior. Identification procedure is the following one:

1. Identify $\varepsilon^{c r}$ from the material peak stress $\sigma_{\max }^{t}$ in tension ( $\varepsilon^{c r}$ is proportional to $\sigma_{\max }^{t}$ ).

2. Identify $\theta^{c r}$ from the material peak stress in compression $\sigma_{\max }^{c}$.

Note that for the forthcoming study, we only deal with tension loading and the identification of parameter $\theta^{c r}$ is not necessary. The response of the discrete model with parameters identified on a concrete behaviour is plotted in figure 3 , for either tension or compression.

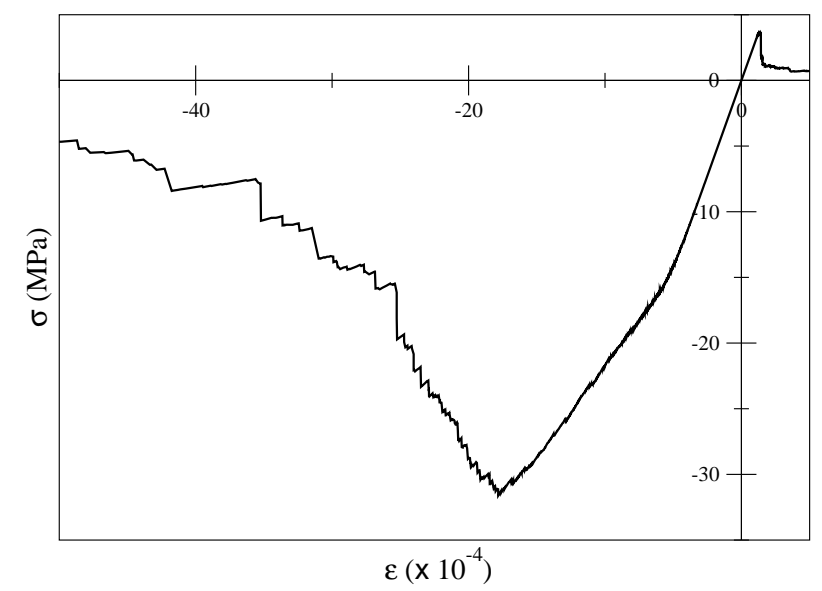

Figure 3: Response of the discrete model in tension and compression $\left(E_{b}=45 \mathrm{GPa}, \alpha=\right.$ $0.74, \varepsilon^{c r}=1.5 \times 10^{-4}$ and $\left.\theta^{c r}=5 \times 10^{-3}\right)$.

We propose next to use this discrete model as a "virtual testing machine". The first part is dedicated to the identification of a parameter of an anisotropic damage model, as the second one is dedicated to the characterization of the biaxial tensile strength of a damaged material.

\section{Identification of a phenomenological model}

\subsection{Anisotropic damage model}

The proposed damage model aims to describe concrete behaviour. It is based on the representation of damage by a tensorial thermodynamic variable, here a second order tensor [Cordebois and Sidoroff, 1982, Ladevèze, 1983, Murakami, 1988, Desmorat et al., 2007]. The chosen state potential is continuously differentiable, a key point to ensure 3D stresses-strains continuity even in a non proportional loading cases. For this model, the Gibbs free enthalpy is split into a deviatoric part and a hydrostatic one [Papa and Taliercio, 1996, Lemaitre et al., 2000]. Using the notation $\langle x\rangle=\max (x, 0)$ for the positive part of a scalar, Gibbs free enthalpy reads:

$$
\begin{aligned}
\rho \psi^{*}= & \frac{1+\nu}{2 E} \operatorname{tr}\left[(\mathbf{1}-\mathbf{D})^{-1 / 2} \boldsymbol{\sigma}^{D}(\mathbf{1}-\mathbf{D})^{-1 / 2} \boldsymbol{\sigma}^{D}\right] \\
& +\frac{1-2 \nu}{6 E}\left[\frac{\langle\operatorname{tr} \boldsymbol{\sigma}\rangle^{2}}{1-\eta D_{H}}+\langle-t r \boldsymbol{\sigma}\rangle^{2}\right]
\end{aligned}
$$

with $E$ and $\nu$ the Young modulus and Poisson ratio of the initially isotropic material, $D_{H}=\frac{1}{3} \operatorname{tr} \mathbf{D}$ and where $(\cdot)^{D}$ denotes the deviatoric part. The parameter $\eta$ represents the material sensitivity 
to hydrostatic stresses, a parameter closely related to strain localisation [Lemaitre et al., 2000]. Note that the dependence of the hydrostatic part on $\operatorname{tr} \mathbf{D}$ has been shown in a previous study by using a discrete model [Delaplace and Desmorat, 2008].

The state laws derive from the state potential (2), the elasticity law reading then

$$
\begin{aligned}
\boldsymbol{\varepsilon}=\rho \frac{\partial \psi^{*}}{\partial \boldsymbol{\sigma}}= & \frac{1+\nu}{E}\left[(\mathbf{1}-\mathbf{D})^{-1 / 2} \boldsymbol{\sigma}^{D}(\mathbf{1}-\mathbf{D})^{-1 / 2}\right]^{D} \\
& +\frac{1-2 \nu}{3 E}\left[\frac{\langle\operatorname{tr} \boldsymbol{\sigma}\rangle}{1-\frac{\eta}{3} \operatorname{tr} \mathbf{D}}-\langle-\operatorname{tr} \boldsymbol{\sigma}\rangle\right] \mathbf{1}
\end{aligned}
$$

The strain energy release rate density, the thermodynamics force associated with the damage $\mathbf{D}$, is gained as [Lemaitre and Desmorat, 2005]

$$
\boldsymbol{Y}=\rho \frac{\partial \psi^{*}}{\partial \mathbf{D}}
$$

Concerning damage, a criterion function $f$ is considered defining the elasticity domain by $f<0$ and damage growth by the consistency condition $f=0$ and $\dot{f}=0$,

$$
f=\hat{\varepsilon}-\kappa(\operatorname{tr} \mathbf{D})
$$

where $\hat{\varepsilon}$ is Mazars equivalent strain [Mazars, 1984, Mazars et al., 1990],

$$
\hat{\varepsilon}=\sqrt{\sum_{I=1}^{3}\left\langle\varepsilon_{I}\right\rangle^{2}}=\sqrt{\langle\varepsilon\rangle_{+}:\langle\varepsilon\rangle_{+}}
$$

built from the positive extensions $\left(\langle\varepsilon\rangle_{+}\right.$is the positive part of the strain tensor in terms of principal values). The function $\kappa$ allowing for modeling both tensile and compressive response of concrete with a single set of material parameters is:

$$
\kappa(\operatorname{tr} \mathbf{D})=a \cdot \tan \left[\frac{\operatorname{tr} \mathbf{D}}{a A}+\arctan \left(\frac{\kappa_{0}}{a}\right)\right]
$$

and

$$
\kappa^{-1}(\operatorname{tr} \mathbf{D})=a A\left[\arctan \left(\frac{\hat{\varepsilon}}{a}\right)-\arctan \left(\frac{\kappa_{0}}{a}\right)\right]
$$

The interested reader may refer to [Desmorat et al., 2007] for a full description of the model and for the numerical implemention in both a local and a nonlocal form. The monotonic response of the model in tension and compression is plotted in figure 4 .

A main feature of the model is, as already stated, the reduced number of material parameters introduced to represent the full 3D anisotropic damage evolution, 6 including the elasticity parameters:

- the elasticity parameters $E, \nu$,

- the damage threshold $\kappa_{0}$,

- the damage parameters $A, a$,

- the parameter of damage sensitivity to hydrostatic stresses $\eta$.

The first five parameters can be easily identified from basic experimental tension and compression tests. On the other hand, last parameter $\eta$ is more subtle to identify, because it is mainly acting on triaxial tension states difficult to represent with an experimental setup for brittle materials. An alternative of experimental tests for these specific identifications is to consider virtual testing (or a "numerical laboratory") for which a numerical model representing the microstructure of the material is used. We propose this alternative by using the discrete model presented above to identify the $\eta$ parameter. 


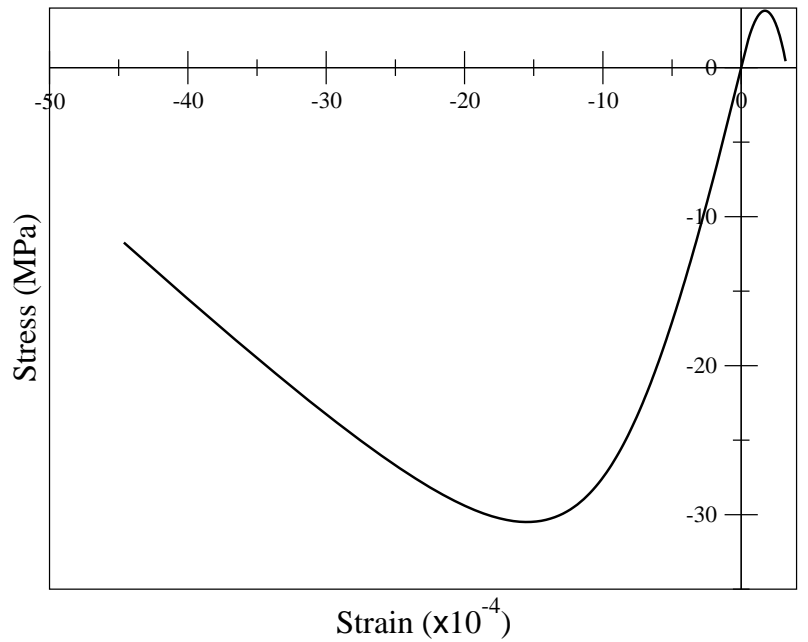

Figure 4: Response of the model in tension and compression $(E=30 \mathrm{GPa}, \nu=0.2$, $\kappa_{0}=5 \times 10^{-5}, a=3 \times 10^{-4}, A=5 \times 10^{3}$ and $\left.\eta=1\right)$.

\subsection{Cross-identification procedure}

The identification strategy of parameter $\eta$ is based on the expression of the damaged -or effectivebulk modulus:

$$
\tilde{K}=\frac{\operatorname{tr} \sigma}{3 \operatorname{tr} \varepsilon}
$$

By using expression (3), one has:

$$
\tilde{K}=\frac{E\left(1-\frac{\eta}{3} \operatorname{tr} \mathbf{D}\right)}{3(1-2 \nu)}=K\left(1-\frac{\eta}{3} \operatorname{tr} \mathbf{D}\right)
$$

where $K$ is the modulus of the virgin material. Considering this last expression, one can propose the following global procedure, based on a succession of uniaxial and triaxial loading tests (figure 5):
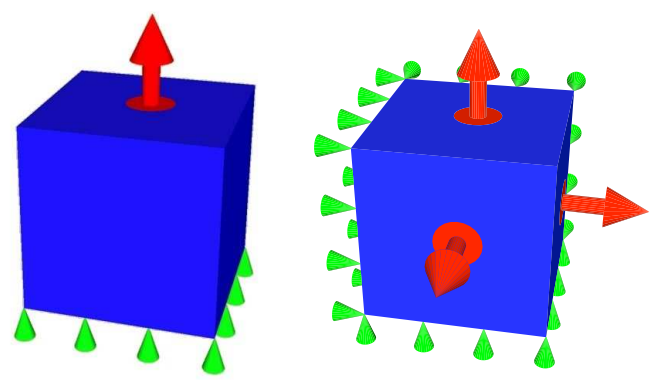

Figure 5: Boundary conditions for uniaxial (left) and triaxial (right) loading states.

- Perform one elastic uniaxial tension test on a cube.

- Measure $E, \nu$ and compute elastic compressibility modulus $K=E /(3(1-2 \nu))$ 
- Perform $n$-nonlinear tritension tests using the discrete model for different loads $q \in\{1, . . n\}$. Proceeds as follows:

1. Apply equally imposed displacements $\bar{u}_{1}^{q}=\bar{u}_{2}^{q}=\bar{u}_{3}^{q}$ on the cube faces and measure the damage bulk modulus $\tilde{K}^{q}$

2. Unload the specimen

3. Apply three elastic uniaxial loads in the three loading directions $x \equiv 1, y \equiv 2, z \equiv 3$.

- Measure damaged Young modulus $\tilde{E}_{1}^{q}, \tilde{E}_{2}^{q}$ and $\tilde{E}_{3}^{q}$

- Compute damage values for each direction

$$
\begin{gathered}
1-D_{1}^{q}=\frac{2(1+\nu)}{E\left(\frac{5}{\tilde{E}_{1}^{q}}-\frac{1}{\tilde{E}_{2}^{q}}-\frac{1}{\tilde{E}_{3}^{q}}-\frac{1}{3 \tilde{K}^{q}}\right)}, \\
1-D_{2}^{q}=\frac{2(1+\nu)}{E\left(-\frac{1}{\tilde{E}_{1}^{q}}+\frac{5}{\tilde{E}_{2}^{q}}-\frac{1}{\tilde{E}_{3}^{q}}-\frac{1}{3 \tilde{K}^{q}}\right)}, \\
1-D_{3}^{q}=\frac{2(1+\nu)}{E\left(-\frac{1}{\tilde{E}_{1}^{q}}-\frac{1}{\tilde{E}_{2}^{q}}+\frac{5}{\tilde{E}_{3}^{q}}-\frac{1}{3 \tilde{K}^{q}}\right)},
\end{gathered}
$$

- Store the set $\left(\tilde{K}^{q}, D_{1}^{q}, D_{2}^{q}, D_{3}^{q}\right)$

- Identify $\eta$ from the linear relation (10), $\tilde{K}^{q} / K=\left(1-\frac{\eta}{3} \operatorname{tr} \mathbf{D}^{q}\right), q \in\{1, . . n\}$.

The identification has been performed on three different samples, with properties presented in table 1.

\begin{tabular}{|c|c|c|}
\hline sample size & number of particles & number of dof \\
\hline $8 \times 8 \times 8$ & 512 & 3072 \\
\hline $16 \times 16 \times 16$ & 4096 & 24576 \\
\hline $32 \times 32 \times 32$ & 32768 & 196608 \\
\hline
\end{tabular}

Table 1: Different samples tested for the identification of parameter $\eta$.

We present here the tritension responses obtained for the 8-cube sample (figure 6). Stressstrain curves are plotted for the three directions of loading and, more important, evolution of the damage moduli $\tilde{E}_{i}, i \in\{x, y, z\}$ are plotted versus $D_{H}$ (upperscripts $q$ corresponding to the maximum applied displacement are omitted next). Stress-strain responses for 16-cube sample and 32-cube sample are plotted in figure 7 .

For successive loading steps, tritension test is stopped and an uniaxial tensile loading is applied elastically in order to obtain the corresponding damage values $D_{i}$. Finally, the evolution of the damages $D_{i}$ versus the effective bulk modulus $\tilde{K}$ is plotted in figure 8. As expected, relationship is linear emphasing the fact that $\eta$ can be considered as a material parameter. The best fitted line is computed and the damage hydrostatic parameter is evaluated from its slope. Finally, parameter $\eta$ is successfully evaluated from the three responses of the three samples:

$$
\eta \approx 1.2
$$

The corresponding crack patterns obtained at the end of the loading are plotted in figure 9 . 

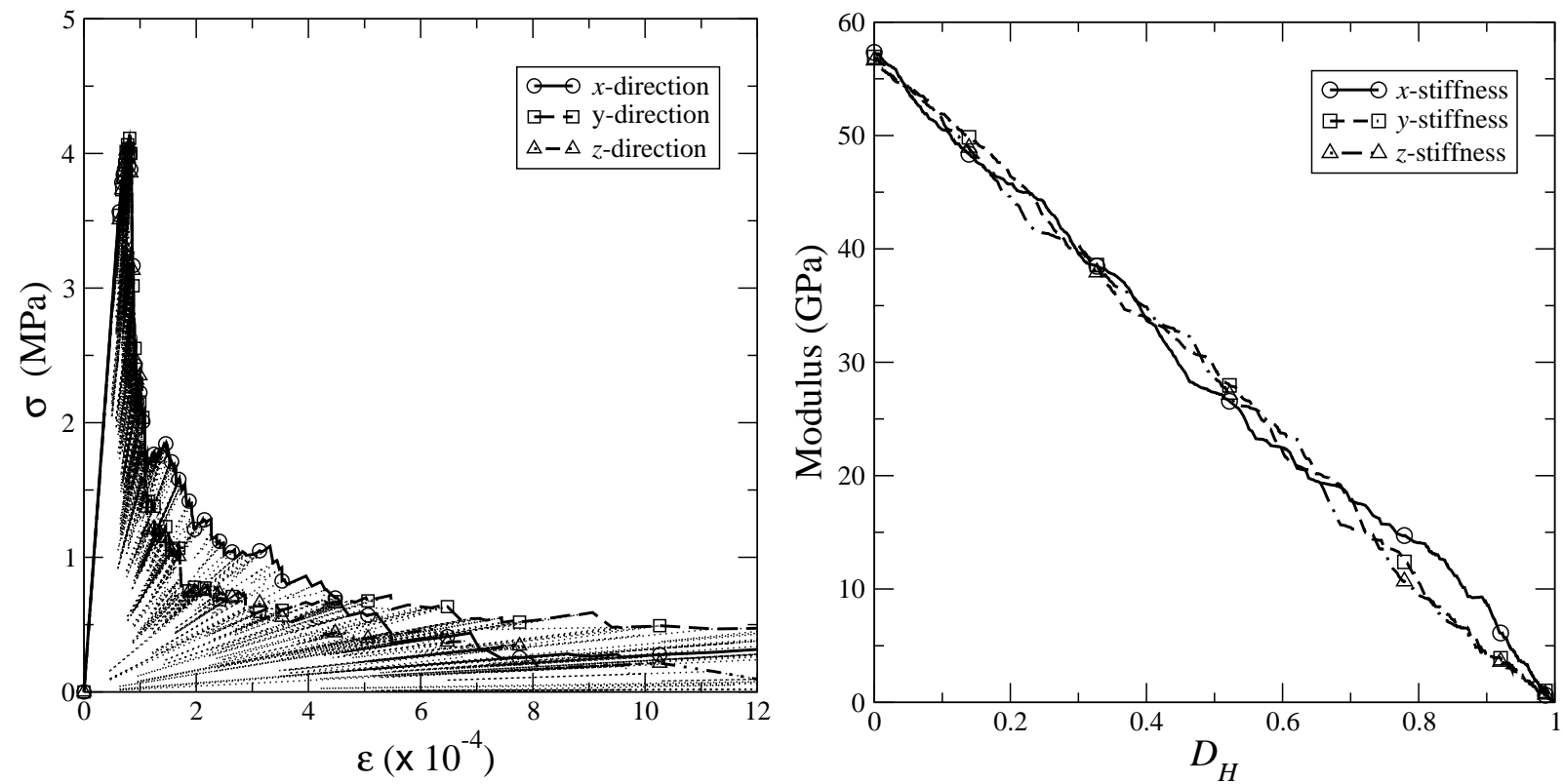

Figure 6: Up: stress-strain responses for the three directions during tri-tension, and bottom: evolution of stiffnesses $\tilde{E}_{x}, \tilde{E}_{y}, \tilde{E}_{z}$.
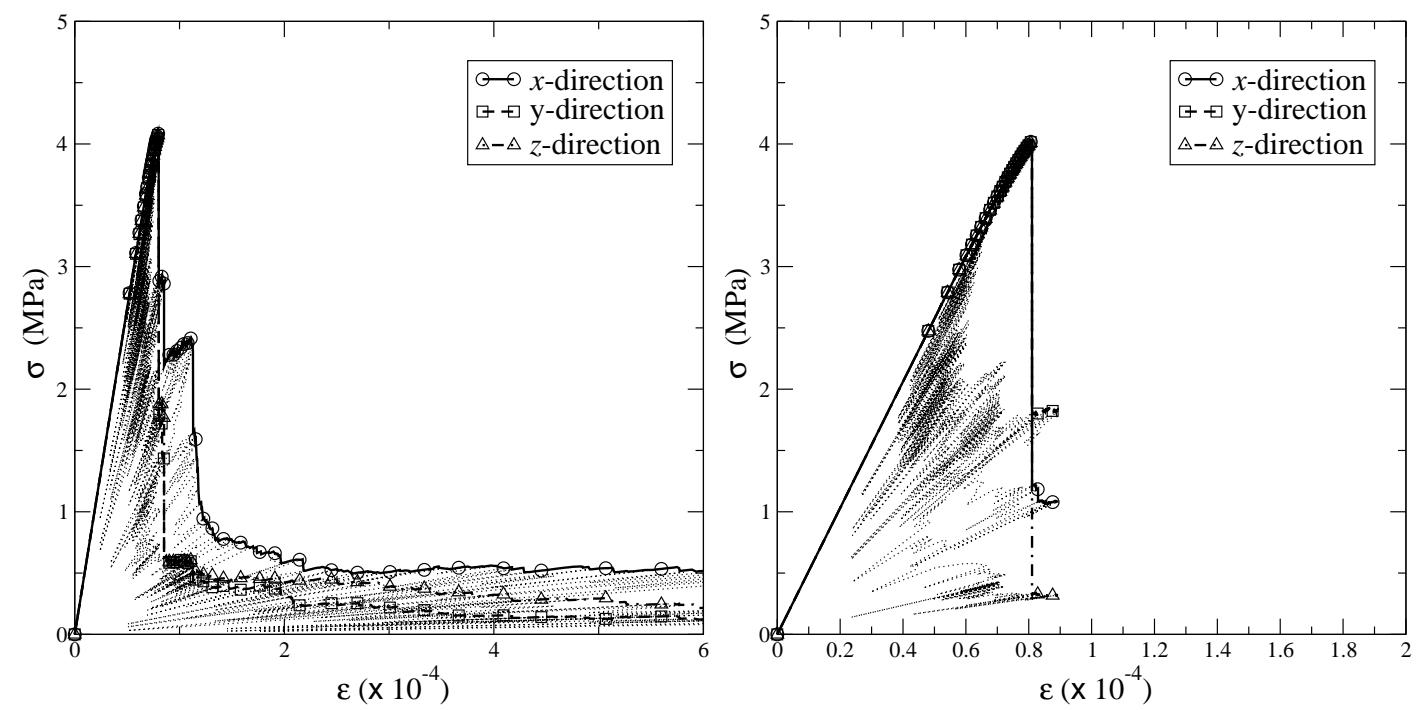

Figure 7: Up: stress-strain responses for the 16-cube sample, and bottom: for the 32-cube sample.

\section{Failure envelope for anisotropically damaged sam- ples}

The determination of a criterion function $f$ is nowadays necessary for delimiting the elasticity domain $(f<0)$ of any plasticity or damage models or to characterize the strength of a material under multiaxial loading. This function can be obtained by considering different proportional loading paths and by fixing an offset. For brittle materials, experiments need a particular setup, 


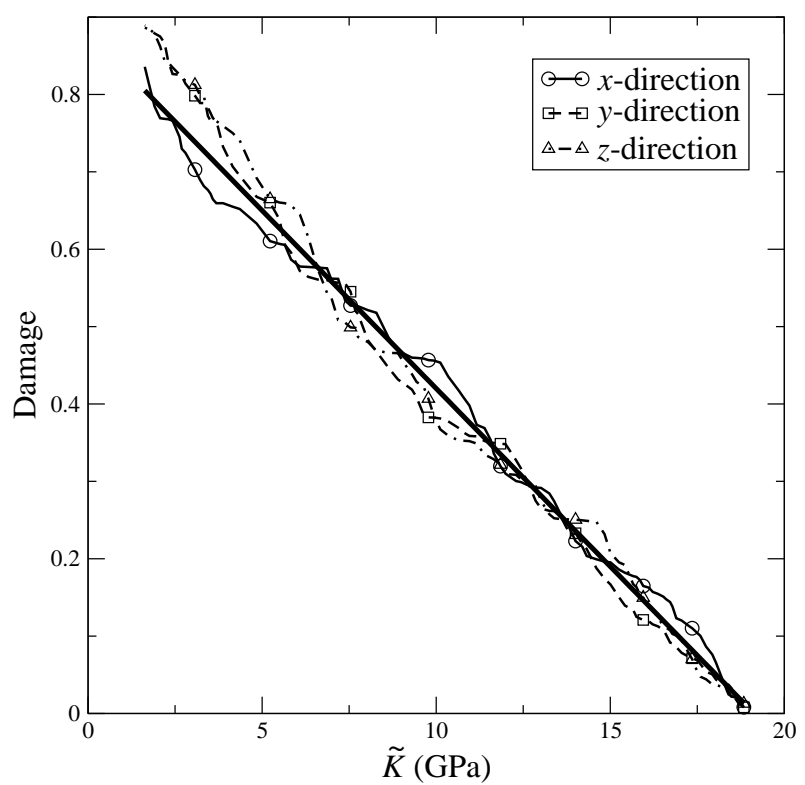

Figure 8: Evolution of damages $D_{i}$ versus $\tilde{K}$ for the 8-cube sample. Bold line is the best fitted line.
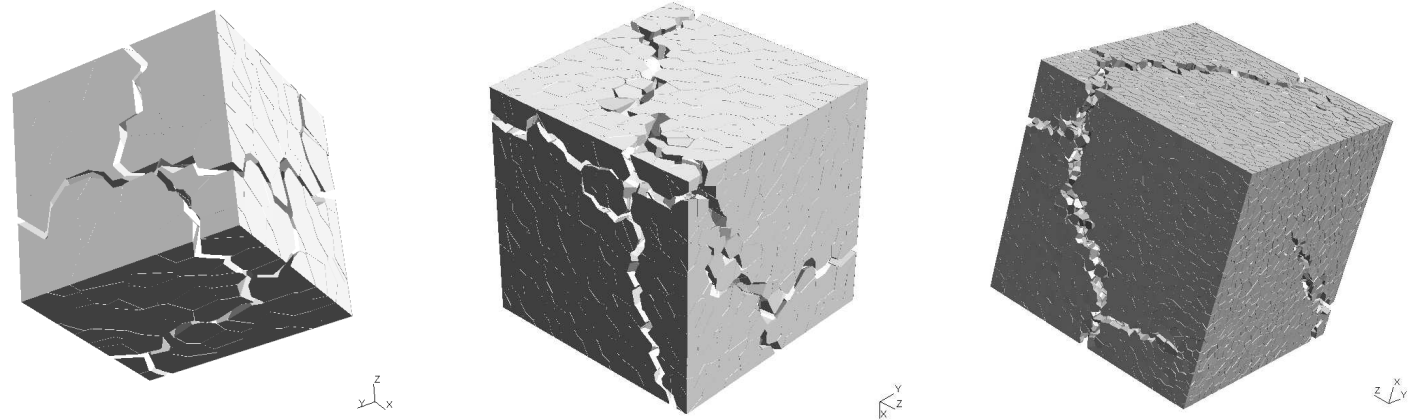

Figure 9: Crack patterns for the three samples (top $8 \times 8 \times 8$, middle $16 \times 16 \times 16$, bottom $32 \times 32 \times 32)$.

especially for biaxial tension-tension loading in order to avoid unstable failure. An example of such a setup is given in [Kupfer and Hilsdorf, 1969, Lee et al., 2004]. In these two studies, the biaxial behavior of concrete is investigated and the criterion functions (for both the elasticity domain and the strength envelope) are determined for initially undamaged specimen. To our knowledge no experiments have been done for initially isotropically and anisotropically damaged samples. This is easily understood considering the difficulty to perform such experiments as for the determination of the $\eta$ parameter in previous part, the experimental procedure is based on the succession of loading (to create an initial (or pre-) damaged state), unloading and reloading (to extract the ultimate strength) with changing boundary conditions. This can be envisaged for compression-compression or compression-tension loading cases, but the tension-tension case will remain difficult to carry out properly. This is why one proposes in this part to perform numerically such tests. This virtual testing is made with discrete models and one focus on the tension-tension region. 


\subsection{Numerical procedure}

We use a $200 \mathrm{~mm} \times 200 \mathrm{~mm} \times 60 \mathrm{~mm}$ square plate form for the numerical sample to mimic the experimental sample used in [Lee et al., 2004]. The number of particles is $20 \times 20 \times 6=2400$, that corresponds to 14400 dof. We apply different ratios $\alpha=f_{2} / f_{1}$ where $f_{1}$ is the applied force in the $x$-direction and $f_{2}$ in the $z$-direction, with a uniform displacement condition on loading faces (figure 11). The chosen ratio are $\alpha=\{0,0.4,0.6,0.8,1 ., 1.25,1.67,2.5, \infty\}$. In our study and contrary to the experimental study of [Kupfer and Hilsdorf, 1969] or [Lee et al., 2004], we do not plot the half part $(1<\alpha<\infty)$ by symmetry with respect to the other one $(0 \leq \alpha \leq 1)$, considering the potential anisotropy of the damage.

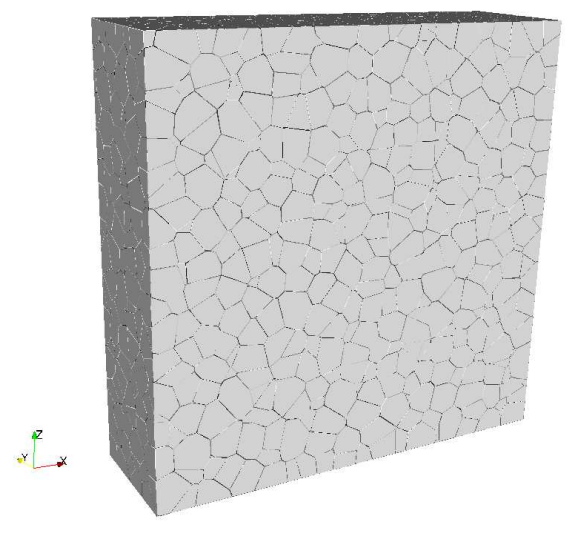

Figure 10: The $20 \times 20 \times 6$-particles sample.

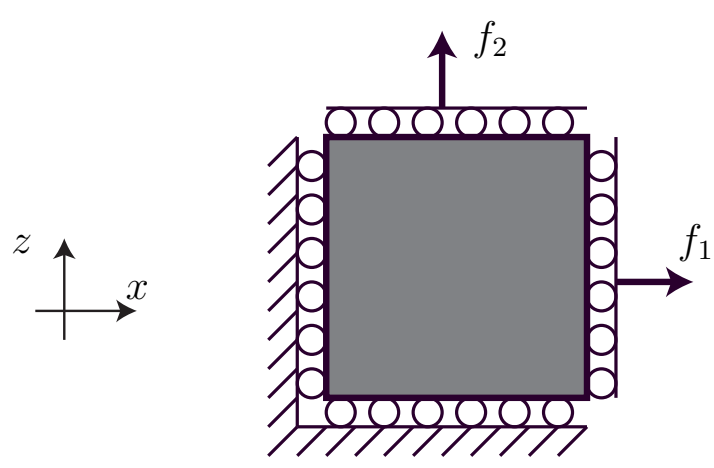

Figure 11: Boundary conditions for the tension-tension specimen.

\subsection{Undamaged failure envelope}

The undamaged failure envelope is shown in figure 12. The numerical curve obtained by simulations is compared to the experimental one obtained by [Lee et al., 2004] (the curve obtained by [Kupfer and Hilsdorf, 1969] is similar). The domain (and the following ones) is presented in a normalized diagram with respect to the uniaxial tension peak stresses $\sigma_{1}^{t 0}$ and $\sigma_{2}^{t 0}$. As expected, the domain numerically obtained is in agreement with the experimental one. In both experimental and numerical cases, it is observed an initial tangent inclination when entering the tension-tension 
region. Note that this inclination, typical of a strain criteria for describing rupture, is rarely represented by analytical criterion functions.

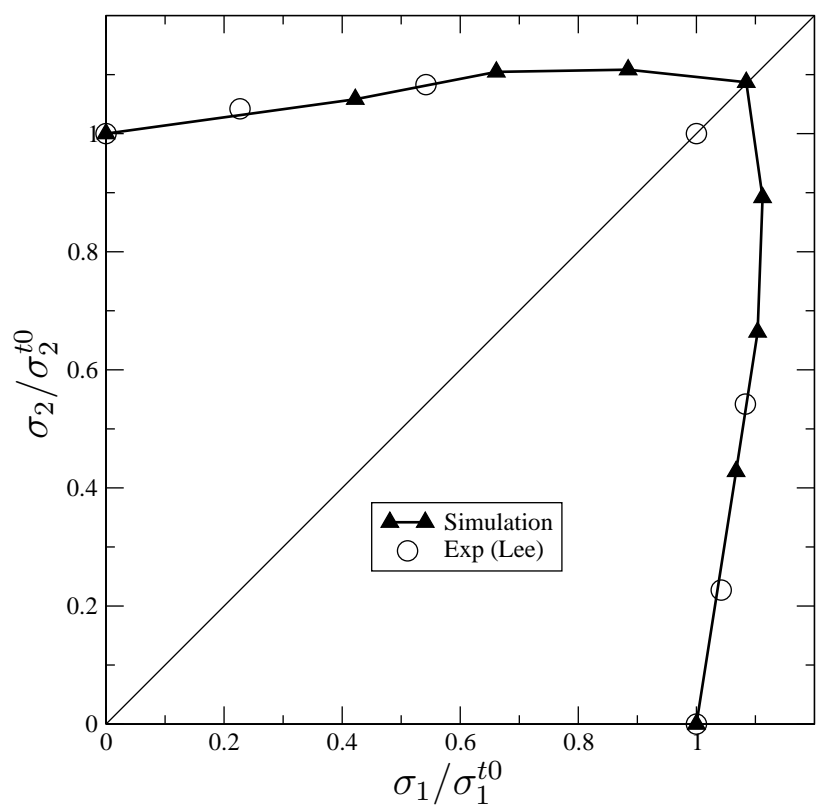

Figure 12: Undamaged failure envelope.

The strain-stress relationship for the different ratios is plotted in figure 13. Results are again really similar to the experimental results of [Lee et al., 2004]. Behavior is quasi-brittle in tension and the linear relationship is naturally obtained.

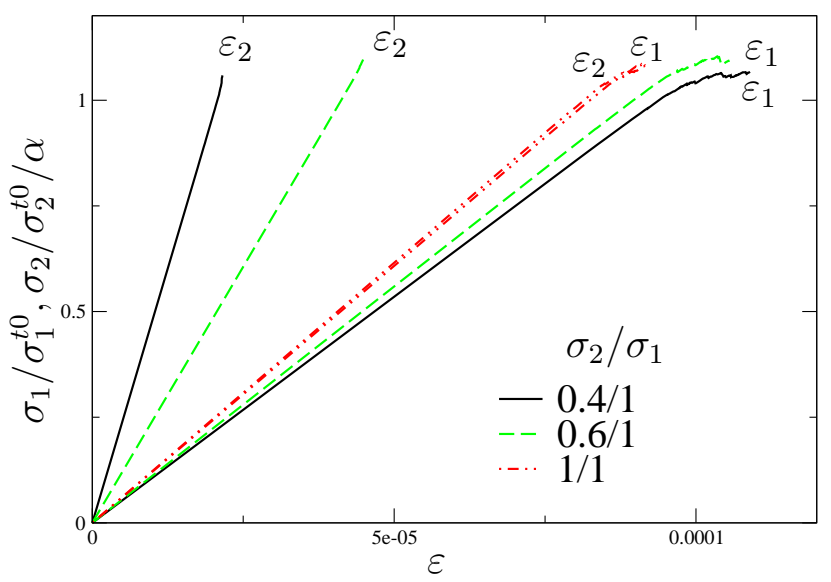

Figure 13: Stress-strain relationship for different ratios.

The last presented results concern the cracking pattern. Figure 14 shows the different cracks orientations for three different loading ratios $\alpha=f_{2} / f_{1}$. Cracks are represented for the discrete model by the drawing of the associated polygon with each breaking link. 

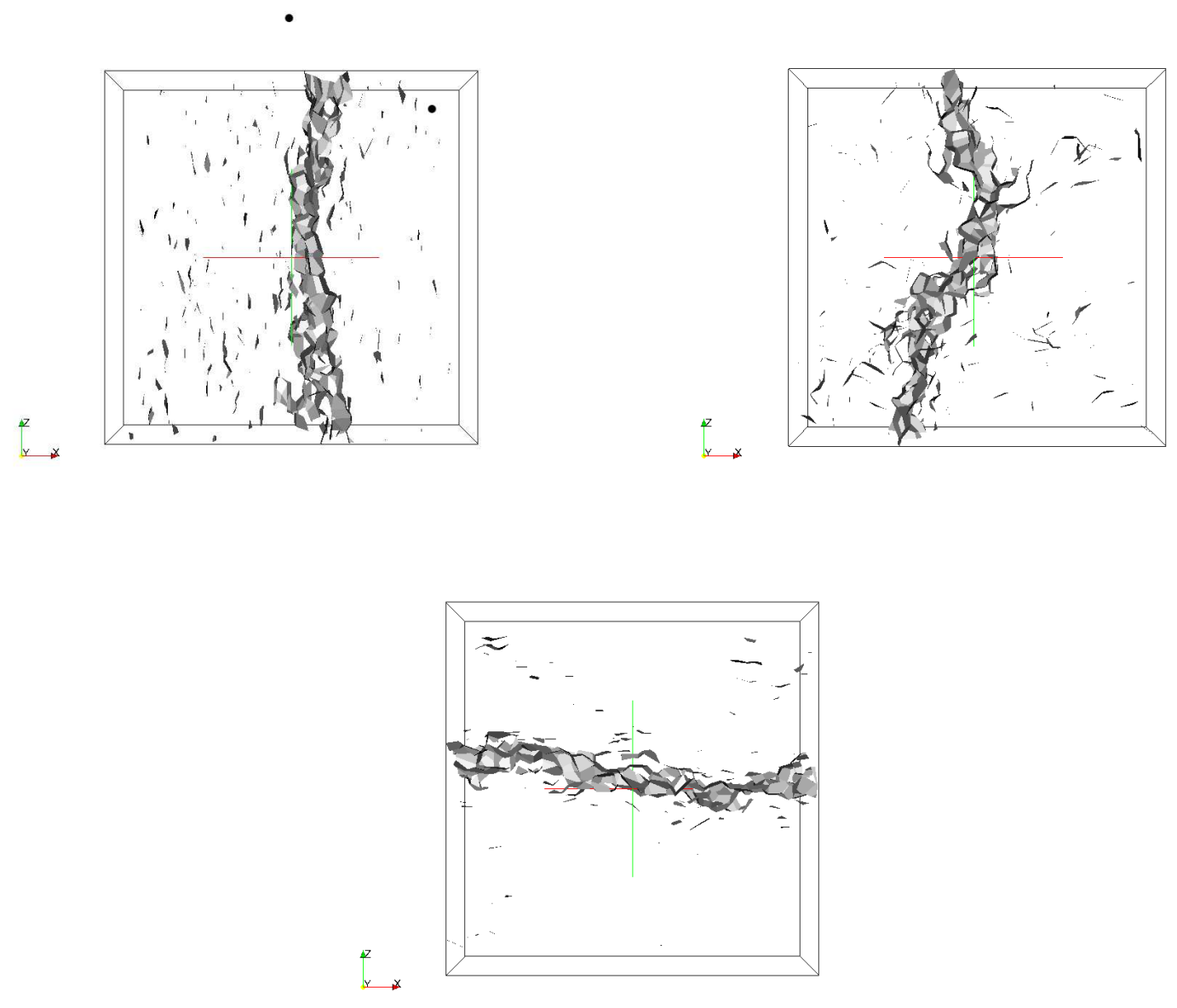

Figure 14: Cracking pattern obtained for the different loading ratios: $\alpha=0$., $\alpha=1$., $\alpha=\infty$.

\subsubsection{Isotropically damaged failure envelope}

We propose in this part to evaluate the evolution of the failure envelope with an initial isotropic damaged or cracking state. This initial damage is obtained by randomly removing bonds between particles in the numerical sample:

- From the initial state, a random value $t_{i}$ picked from a uniform distribution between 0 and 1 is ascribed to each bond $i$.

- For a given threshold $t_{c}$, a link $i$ is removed if $t_{i}>t_{c}$.

- Furthermore, to control the orientation of damage, a link $i$ is removed only if the normal vector $\vec{n}_{i}$ of the corresponding facet (i.e. the common polygon of the two neighboring particles) verifies $\vec{n}_{i} \cdot \vec{x}>\vec{n}_{i} \cdot \vec{y}$ or $\vec{n}_{i} \cdot \vec{z}>\vec{n}_{i} \cdot \vec{y}$.

Table 2 gives the evolution of the normalized apparent stiffnesses $E_{x} / E_{x}^{0}$ and $E_{z} / E_{z}^{0}$ after bonds removing for a sample with initial stiffnesses $E_{x}^{0}=E_{z}^{0}=30.0 \mathrm{GPa}$.

Figure 15 shows the evolution of the stiffnesses and the evolution of the peak stresses for a uniaxial test with respect to the threshold values $\left(t_{c}=t_{c}^{x}=t_{c}^{z}\right)$.

The evolution of the new failure envelope after pre-damaging is plotted in figure 17 . Not surprisingly, the different envelopes are similar. The main effect is the evolution from an acute 


\begin{tabular}{|c|c|c|c|c|c|c|}
\hline$t_{c}$ & 0.9 & 0.8 & 0.7 & 0.6 & 0.5 & 0.4 \\
\hline$E_{x} / E_{x}^{0}$ & 0.839 & 0.674 & 0.525 & 0.394 & 0.286 & 0.190 \\
\hline$E_{z} / E_{z}^{0}$ & 0.844 & 0.680 & 0.531 & 0.412 & 0.293 & 0.197 \\
\hline
\end{tabular}

Table 2: Evolution of the effective stiffnesses for different threshold values.
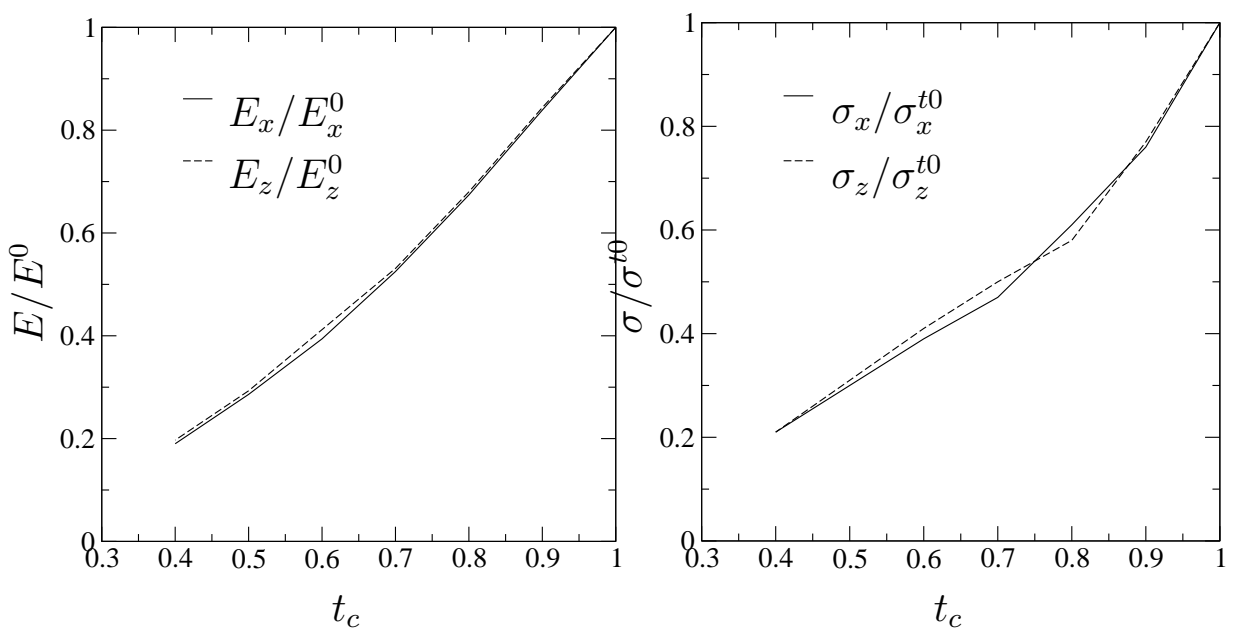

Figure 15: Up: evolution of the normalized stiffness $E_{x} / E_{x}^{0}$ and $E_{z} / E_{z}^{0}$ and bottom: evolution of the normalized peak stresses $\sigma_{x} / \sigma_{x}^{t 0} \equiv \sigma_{1} / \sigma_{1}^{t 0}$ and $\sigma_{z} / \sigma_{z}^{t 0} \equiv \sigma_{2} / \sigma_{2}^{t 0}$ for initial isotropic damage.

shape to an (slightly) obtuse one, with a decrease of the bitension peak stress with respect to the uniaxial one. The initial tangent inclination observed for the undamaged state decreases and becomes negative at high pre-damage levels. Note the asymmetric shape of some envelopes due to the non purely isotropic repartition of damage, asymmetry which can not be revealed if symmetrically conditions are applied to plot the envelope. The failure envelopes normalized to each damaged peak stress are shown in figure 17. The evolution of the envelope shape is clearly visible.

\subsubsection{Anisotropically damaged failure envelope}

In this part, anisotropic pre-damage is considered. We limit our study to initial damage in the $z$-direction. We proceed as for the initial isotropic damage, i.e. link $i$ is removed if its associated random value $t_{i}>t_{c}^{z}$ and if $\vec{n}_{i} \cdot \vec{z}>\vec{n}_{i} \cdot \vec{x}$ and $\vec{n}_{i} \cdot \vec{z}>\vec{n}_{i} \cdot \vec{y}$. Table 3 gives the evolution of the normalized apparent stiffness $E_{x} / E_{x}^{0}$ and $E_{z} / E_{z}^{0}$ for a sample with initial stiffnesses $E_{x}^{0}=E_{z}^{0}=$ $30.0 \mathrm{GPa}$, and the evolution of the normalized stiffnesses and normalized peak stresses are plotted in figure 18. Note the small but non null reduction of $E_{x}$ : removed links are mainly oriented along $z$-axis, but the $x$-component of the normal vector is not null.

Finally, failure envelopes for damage anisotropy is plotted in figure 19. In this normalized diagram, the evolution is similar to the isotropic one. Again, the initial tangent inclination decreases for direction 2. In a normalized diagram with respect to the damaged uniaxial peak stresses (figure 20), the envelopes become symmetric with respect to the bisecting line $\sigma_{1}=\sigma_{2}$ and look like the envelopes obtained for the initial isotropic damaged states. 


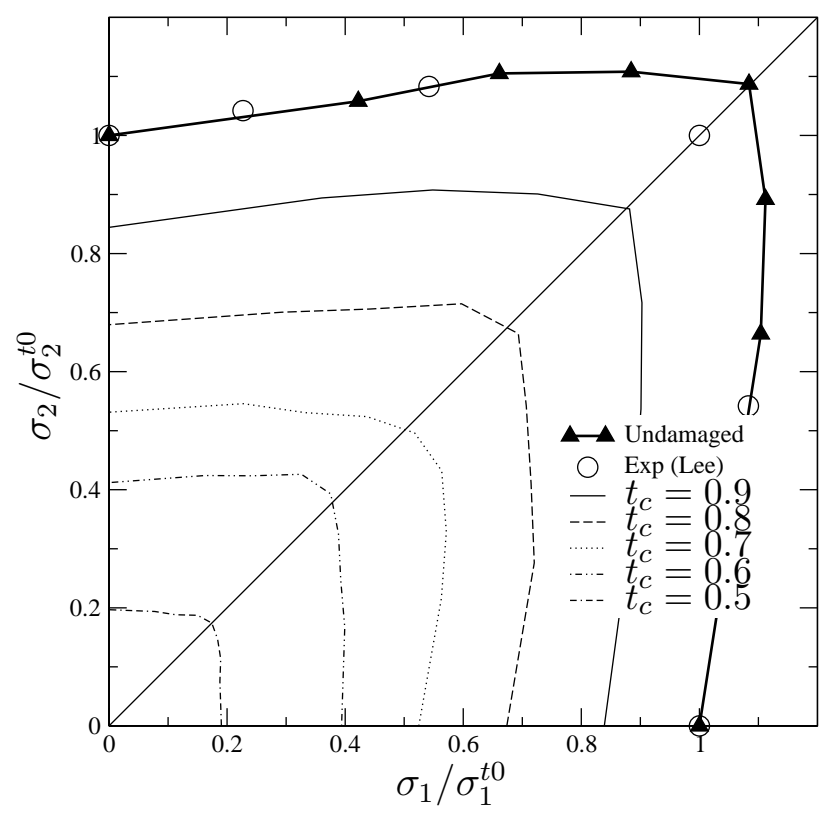

Figure 16: Iso-damaged failure envelopes normalized to undamaged uniaxial peak stresses.

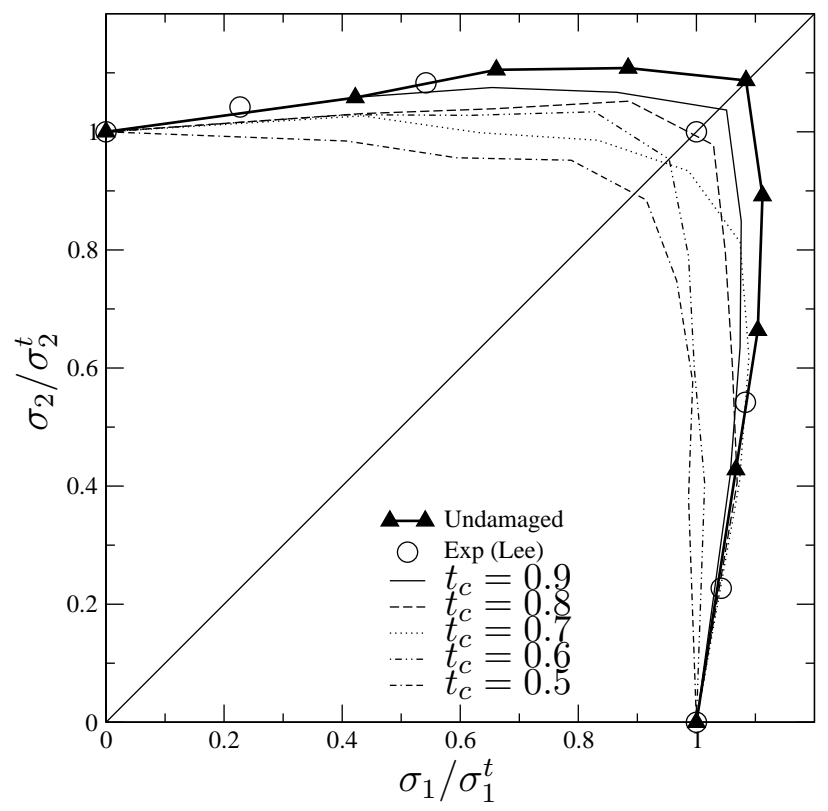

Figure 17: Iso-damaged failure envelopes normalized to damaged uniaxial peak stresses.

\section{Conclusion}

We have proposed in this paper the use of a discrete particle model as a complimentary numerical or virtual testing in conditions where experiments are difficult to perform (multiaxial tensile loading on quasi-brittle materials, repetition of elastic loading on the same sample but in different directions). In particular, the effect of damage anisotropy is investigated. The identification of a specific parameter of a phenomenological anisotropic damage model has been successfully performed, noting that the first five parameters of the model are easily identified from classi- 


\begin{tabular}{|c|c|c|c|}
\hline$t_{c}^{z}$ & 0.9 & 0.7 & 0.5 \\
\hline$E_{x} / E_{x}^{0}$ & 0.979 & 0.926 & 0.877 \\
\hline$E_{z} / E_{z}^{0}$ & 0.866 & 0.600 & 0.392 \\
\hline
\end{tabular}

Table 3: Evolution of the effective stiffness for different threshold values for initial anisotropic damage.
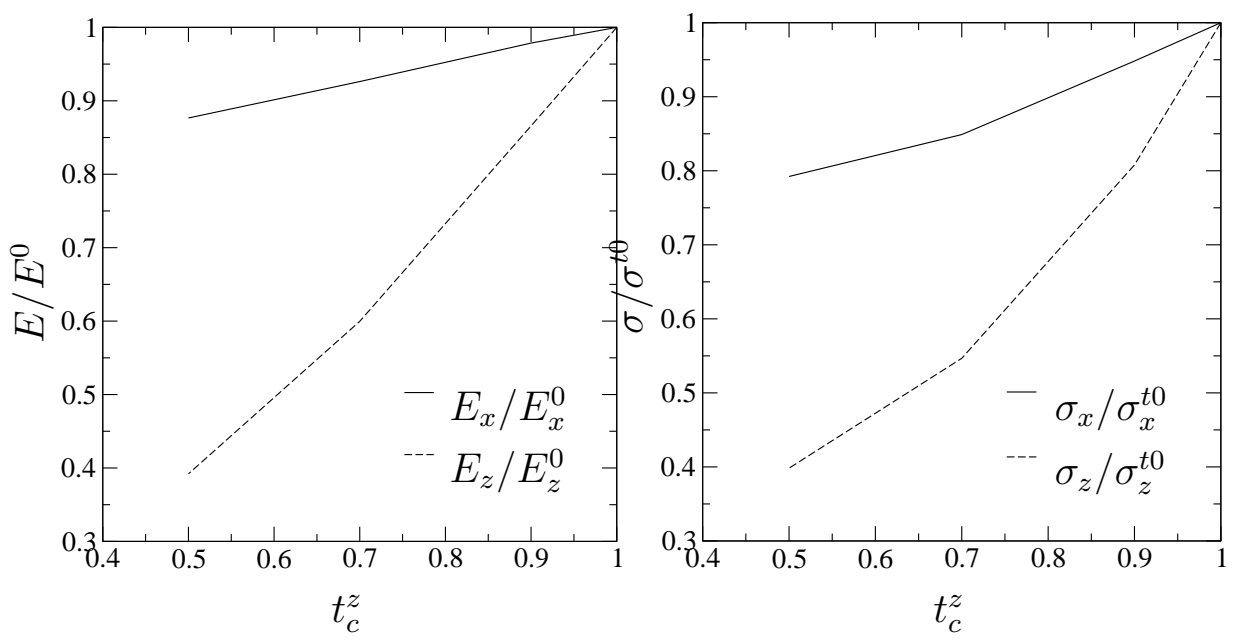

Figure 18: Up: evolution of the normalized stiffness $E_{x} / E_{x}^{0}$ and $E_{z} / E_{z}^{0}$ and bottom: evolution of the normalized peak stresses $\sigma_{x} / \sigma_{x}^{t 0} \equiv \sigma_{1} / \sigma_{1}^{t 0}$ and $\sigma_{z} / \sigma_{z}^{t 0} \equiv \sigma_{2} / \sigma_{2}^{t 0}$ for initial anisotropic damage.

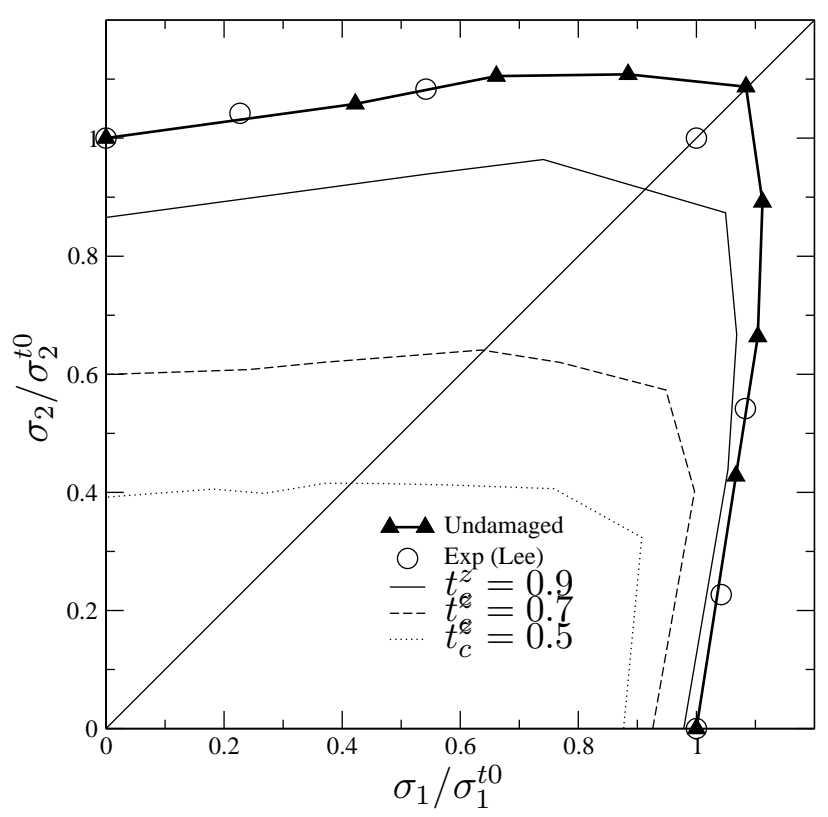

Figure 19: Aniso-damaged criterion functions normalized to undamaged uniaxial peak stresses. 


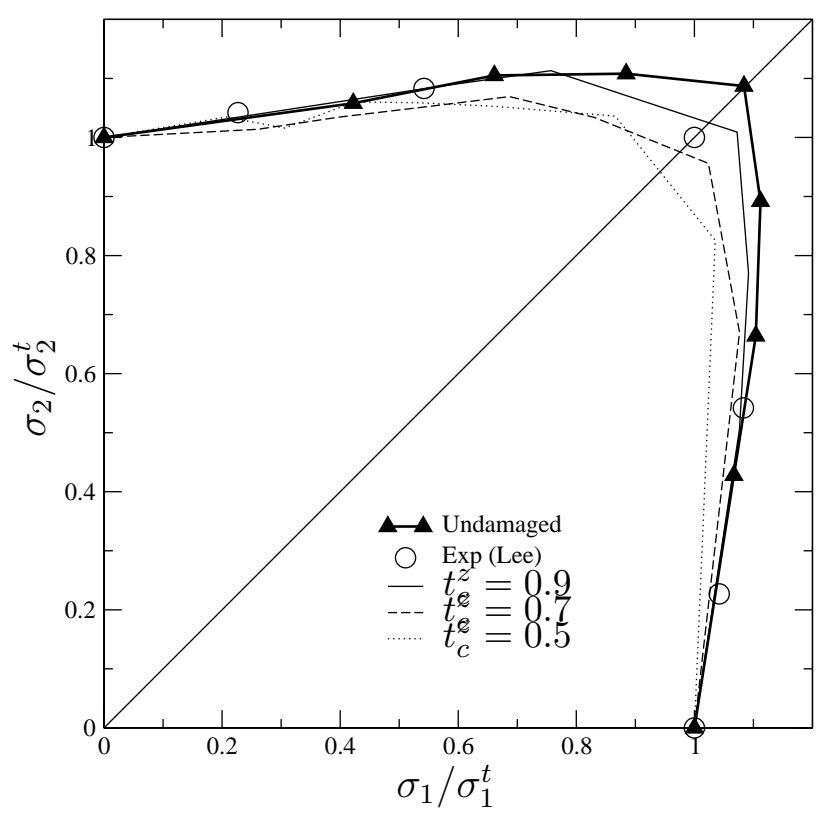

Figure 20: Aniso-damaged criterion functions normalized to damaged uniaxial peak stresses.

cal experimental tension-compression tests. The parameter of damage sensitivity to hydrostatic stresses, $\eta$, is gained from a proposed identification procedure, based on a succession of loading and unloading tension tests with varying boundary conditions. This procedure can not be envisaged experimentally for quasi-brittle materials. The parameter is identified to be $\eta \approx 1.2$ for quasi-brittle materials, value quite different to the values obtained for plastic metallic materials for which $\eta \in[2,3]$ [Lemaitre et al., 2000] and which corresponds to a cross-identification with the discrete model.

One has proposed to characterize the evolution of the failure envelope for different initial damaged (pre-damaged) states. The failure envelope for quasi-brittle material has been experimentally obtained for brittle material, but only for initial undamaged state. The discrete model allows a fine control of the initial damage, isotropic or not. After a comparison between experimental and numerical failure envelopes for undamaged state, we study the evolution of the envelope with damage evolution. We show that the initial acute shape of the envelope becomes slightly obtuse one, both for isotropic and anisotropic damaged states.

This first attempt shows the interest of discrete element model for characterizing the multiaxial tensile strength of initially undamaged or initially damaged materials. 


\section{References}

[Bolander and Saito, 1998] Bolander, J. E. and Saito, S. (1998). Fracture analysis using spring networks with random geometry. Engineering Fracture Mechanics, 61:569-591.

[Cordebois and Sidoroff, 1982] Cordebois, J. P. and Sidoroff, J. P. (1982). Endommagement anisotrope en élasticité et plasticité. J.M.T.A., Numéro spécial, pages 45-60.

[Cundall and Strack, 1979] Cundall, P. A. and Strack, O. D. L. (1979). A discrete numerical model for granular assemblies. Géotechnique, 29:47-65.

[D'Addetta et al., 2002] D'Addetta, G. A., Kun, F., and Ramm, E. (2002). On the application of a discrete model to the fracture process of cohesive granular materials. Granular Matter, 4:77-90.

[Delaplace and Desmorat, 2008] Delaplace, A. and Desmorat, R. (2008). Discrete 3d model as complimentary numerical testing for anisotropic damage. International Journal of Fracture, DOI : 10.1007/s10704-008- 9183-9:--.

[Delaplace and Ibrahimbegovic, 2006] Delaplace, A. and Ibrahimbegovic, A. (2006). Performance of time-stepping schemes for discrete models in fracture dynamic analysis. International Journal for Numerical Methods in Engineering, 65:1527-1544.

[Desmorat et al., 2007] Desmorat, R., Gatuingt, F., and Ragueneau, F. (2007). Nonlocal anisotropic damage model and related computational aspects for quasi-brittle materials. Eng. Fracture Mechanics, 74:1539-1560.

[Desmorat and Otin, 2007] Desmorat, R. and Otin, S. (2007). Cross-identification isotropic/anisotropic damage and application to anisothermal structural failure. Engineering Fracture Mechanics, to be published.

[Fayolle et al., 2007] Fayolle, X., Calloch, S., and Hild, F. (2007). Controlling testing machines with digital image correlation. Experimental Techniques, pages 57-63.

[Garboczi et al., 2004] Garboczi, E. J., Bullard, J. W., and Bentz, D. P. (2004). Virtual testing of cement and concrete-usa 2004. Concrete International, 26:33-37.

[Gonzlez and LLorca, 2007] Gonzlez, C. and LLorca, J. (2007). Virtual fracture testing of composites: A computational micromechanics approach. Engineering Fracture Mechanics, 74:11261138.

[Herrmann and Roux, 1990] Herrmann, H. J. and Roux, S. (1990). Statistical models for the fracture of disordered media. Elsevier Science Publishers, Amsterdam.

[Kupfer and Hilsdorf, 1969] Kupfer, H. and Hilsdorf, H. (1969). Behavior of concrete under biaxial stress. ACI J. Proc., pages 656-666.

[Ladevèze, 1983] Ladevèze, P. (1983). On an anisotropic damage theory. In Proc. CNRS Int. Coll. 351 Villars-de-Lans, Failure criteria of structured media, J. P. Boehler ed. 1993, pages $355-363$.

[Lee et al., 2004] Lee, S.-K., Song, Y.-C., and Han, S.-H. (2004). Biaxial behavior of plain concrete of nuclear containment building. Nuclear Engineering and Design, 227:143-153.

[Lemaitre and Desmorat, 2005] Lemaitre, J. and Desmorat, R. (2005). Engineering Damage Mechanics : Ductile, Creep, Fatigue and Brittle Failures. Springer.

[Lemaitre et al., 2000] Lemaitre, J., Desmorat, R., and Sauzay, M. (2000). Anisotropic damage law of evolution. Eur. J. Mech., A/ Solids, 19:187-208. 
[Mazars, 1984] Mazars, J. (1984). Application de la mécanique de l'endommagement au comportement non linéaire et à la rupture du béton de structure. $\mathrm{PhD}$ thesis, Thè se d'Etat Université Paris 6.

[Mazars et al., 1990] Mazars, J., Berthaud, Y., and Ramtani, S. (1990). The unilateral behavior of damage concrete. Eng. Fract. Mech., 35:629-635.

[Moes et al., 1999] Moes, N., Dolbow, J., and Belytschko, T. (1999). A finite element method for crack growth without remeshing. International Journal for Numerical Methods in Engineering, 46:131-150.

[Moukarzel and Herrmann, 1992] Moukarzel, C. and Herrmann, H. J. (1992). A vectorizable random lattice. J. Stat. Phys., 68:911-923.

[Murakami, 1988] Murakami, S. (1988). Mechanical modeling of material damage. J. App. Mech., $55: 280-286$.

[Papa and Taliercio, 1996] Papa, E. and Taliercio, A. (1996). Anisotropic damage model for the multi-axial static and fatigue behaviour of plain concrete. Engineering Fracture Mechanics, 55:163-179.

[Potyondy and Cundall, 2004] Potyondy, D. O. and Cundall, P. A. (2004). A bonded-particle model for rock. International Journal of Rock Mechanics and Mining Sciences, 41(8):13291364 .

[Schlangen and Garboczi, 1997] Schlangen, E. and Garboczi, E. J. (1997). Fracture simulations of concrete using lattice models: Computational aspects. Eng. Fracture Mech., 57(2/3):319-332.

[Schlangen and Van Mier, 1992] Schlangen, E. and Van Mier, J. G. M. (1992). Experimental and numerical analysis of micromechanisms of fracture of cement-based composites. Cement and Concrete Composites, 14:105-118.

[Sun et al., 2007] Sun, Z., Garboczi, E. J., and Shah, S. P. (2007). Modeling the elastic properties of concrete composites: Experiment, differential effective medium theory, and numerical simulation. Cement and Concrete Composites, 29:22-38.

[Sutton et al., 2000] Sutton, M., McNeill, S., Helm, J., and Chao, Y. (2000). Advances in twodimensional and three-dimensional computer vision. Photomechanics, springer, page 323?372.

[Van Mier et al., 2002] Van Mier, J. G. M., Van Vliet, M. R. A., and Wang, T. K. (2002). Fracture mechanisms in particle composites: statistical aspects in lattice type analysis. Mech. Mater, 34:705-724.

[Wells and Sluys, 2000] Wells, G. and Sluys, L. (2000). Three-dimensional embedded discontinuity model for brittle fracture. International Journal of Solids and Structures, 38:897-913. 